\title{
Fatores associados à autopercepção de saúde entre idosos de grupos comunitários
}

\section{Factors associated with self-rated health in older adults from community groups}

\section{Factores asociados con la autoimagen de salud entre mayores de grupos comunitarios}

\section{Camila Teixeira Vaz iD}

Universidade Federal de Juiz de Fora - Governador Valadares (MG) - Brasil

Nizia Araujo Vieira Almeida (iD

Universidade Federal de Juiz de Fora - Governador Valadares (MG) - Brasil

Regina Gendzelevski Kelmann (iD

Universidade Federal de Juiz de Fora - Governador Valadares (MG) - Brasil

Andreia Cristiane Carrenho Queiroz

Universidade Federal de Juiz de Fora - Governador Valadares (MG) - Brasil

Maria Cristina de Albuquerque Barbosa iD

Universidade Federal de Juiz de Fora - Governador Valadares (MG) - Brasil

Clarice Lima Alvares da Silva iD

Universidade Federal de Juiz de Fora - Governador Valadares (MG) - Brasil

\section{RESUMO}

Objetivo: Investigar a associação entre a autopercepção negativa de saúde e características individuais entre idosos de grupos comunitários do Sudeste do Brasil. Métodos: Trata-se de estudo transversal, realizado entre os anos 2014 a 2017, com amostra de 157 idosos participantes de 2 grupos de convivência de dispositivos sociais e/ou religiosos da cidade de Juiz de Fora, Minas Gerais, Brasil. Por meio de um questionário estruturado, baseado em questões utilizadas em inquérito populacional e de aferição de medidas antropométricas de peso, altura e circunferência da cintura (CC), avaliou-se a autopercepção de saúde (variável resposta) por meio da seguinte pergunta: o(a) senhor(a) diria que sua saúde está: ruim, razoável, boa ou muito boa? As respostas foram categorizadas em autopercepção de saúde negativa (ruim e razoável) e positiva (boa e muito boa). Realizaram-se estatísticas descritivas, testes Mann-Whitney, Fisher e qui-quadrado e regressão logística multivariada. Resultados: A proporção de autopercepção negativa de saúde foi de 32,5\%. A autopercepção negativa de saúde associou-se à menor renda (OR=5,02; IC95\%: 2,08 - 12,08), à inatividade física $(O R=3,51$; IC95\%: 1,26 - 9,78) e à presença de duas ou mais doenças $(O R=4,96$; IC95\%: 2,10 - 11,72), independentemente da idade e do sexo. Conclusão: A autopercepção negativa de saúde associou-se à menor renda familiar, à inatividade física e à presença de duas ou mais doenças associadas.

Descritores: Idoso; Autopercepção; Fatores de Risco; Estudos Transversais.

\section{ABSTRACT}

Objective: To investigate the association between negative self-perception of health and individual characteristics in older adults from community groups in Southeastern Brazil. Methods: This cross-sectional study was carried out between 2014 and 2017 with a sample of 157 older adults from 2 social groups held by social and/or religious services in the city of Juiz de Fora, Minas Gerais, Brazil. A structured questionnaire containing questions used in population-based surveys was used and anthropometric measurements of weight, height and waist circumference (WC) were taken. Self-rated health (the outcome variable) was assessed using the following question: would you say your health is bad, fair, good, or very good? The answers were categorized into negative self-perception (poor and fair) and positive self-perception (good and very good). Descriptive statistics, Mann-Whitney test, Fisher's test, Chi-squared test, and multivariate logistic regression were used. Results: The rate of negative self-perception of health was $32.5 \%$. Negative self-perception of health was associated with lower levels of income (OR=5.02; $95 \% \mathrm{Cl}$ : 2.08 12.08), physical inactivity (OR=3.51; 95\% Cl: 1.26 - 9.78), and presence of two or more diseases (OR=4.96, 95\% Cl: 2.10 - 11.72), 
regardless of age and sex. Conclusion: Negative self-perception of health was associated with lower levels of income, physical inactivity and presence of two or more associated diseases.

Descriptors: Aged; Self Concept; Risk Factors; Cross-Sectional Studies.

\section{RESUMEN}

Objetivo: Investigar la asociación entre la auto percepción negativa de salud y las características individuales entre mayores de grupos comunitarios del Sudeste de Brasil. Métodos: Se trata de un estudio transversal realizado entre los años 2014 y 2017 con la muestra de 157 mayores participantes de 2 grupos de convivencia de dispositivos sociales y/o religiosos de la ciudad de Juiz de Fora, Minas Gerais, Brasil. A través de un cuestionario estructurado basado en las preguntas utilizadas en encuesta poblacional y tras la verificación de las medidas antropométricas de peso, altura y circunferencia de la cintura (CC), se evaluó la auto percepción de la salud (variable respuesta) a través de la siguiente pregunta: ¿En su opinión su salud está mala, mediana, buena o muy buena? Las respuestas han sido categorizadas en auto percepción de salud negativa (mala y mediana) y positiva (buena y muy buena). Se realizaron las estadísticas descriptivas, las pruebas de Mann-Whitney, Fisher y chi-cuadrado y la regresión logística multivariada. Resultados: La proporción de la auto percepción negativa de salud ha sido del 32,5\%. La auto percepción negativa de salud se asoció con la menor renta (OR=5,02; IC95\%: 2,08 - 12,08), la inactividad física (OR=3,51; IC95\%: 1,26 - 9,78) y la presencia de dos o más enfermedades (OR=4,96; IC95\%: 2, 10 - 11,72), independientemente de la edad y del sexo. Conclusión: La auto percepción negativa de salud se asoció con la menor renta familiar, la inactividad física y la presencia de dos o más enfermedades asociadas.

Descriptores: Anciano; Autoimagen; Factores de Riesgo; Estudios Transversales.

\section{INTRODUÇÃO}

O envelhecimento populacional é uma conquista mundial e reflete, de forma geral, a melhora das condições de vida e de saúde vivenciadas pelos indivíduos. Nos países desenvolvidos, essa mudança no perfil etário ocorreu de forma gradual ao longo dos dois últimos séculos ${ }^{(1,2)}$. Por outro lado, nos países em desenvolvimento, como 0 Brasil, esse fenômeno se deu de forma rápida e intensa, gerando grandes desafios para as políticas públicas ${ }^{(1-3)}$.

As pessoas idosas convivem, em sua maioria, com doenças crônicas e múltiplas, que levam ao consumo de elevado número de medicamentos, à incapacidade funcional, à fragilidade e outras complicações, favorecendo as internações hospitalares recorrentes e longas. Para além dessas características, o processo de envelhecimento também é afetado por condições, estilos de vida e fatores ambientais e emocionais, sendo agravado por um cenário de escassez de recursos sociais e financeiros da pessoa idosa ${ }^{(1,4,5)}$. Nesse contexto, torna-se essencial uma avaliação multidimensional para a compreensão do envelhecimento e das suas demandas de cuidado em nível individual e populacional, contribuindo para o reconhecimento dos diversos fatores que influenciam a qualidade de vida e o bem-estar desse grupo específico(6).

Nesse sentido, a autopercepção é definida como conjunto de atributos ou características pelos quais o indivíduo descreve a si mesmo, sendo a mesma construída a partir de experiências e interpretações próprias ${ }^{(7)}$. Quando empregada na descrição do estado geral de saúde, a autopercepção ou autoavaliação é um indicador válido de qualidade de vida, morbidade e funcionalidade, e um bom preditor de mortalidade ${ }^{(8)}$, de forma que seu uso tem se ampliado, nas últimas décadas, como importante marcador de saúde nessa faixa etária ${ }^{(4,9,10)}$, por ser simples, de baixo custo e com elevado potencial de aplicabilidade na triagem de risco para controle e redução da vulnerabilidade da pessoa idosa ${ }^{(11)}$.

A autopercepção de saúde deve ser avaliada de forma multiprofissional, uma vez que as percepções do próprio envelhecer influenciam e são influenciadas por dimensões psicológicas, físicas, funcionais e clínicas, além do fato de que o envelhecimento populacional já é uma realidade para os diferentes níveis de atenção à saúde(11).

Assim, considerando-se os múltiplos determinantes da autopercepção de saúde entre idosos, justifica-se a necessidade de estudos frequentes que investiguem o perfil dessa avaliação e seus fatores associados em nível nacional, regional e mesmo local, como ferramenta de vigilância e elemento indispensável ao planejamento de políticas públicas e programas de intervenção voltadas a esse grupo. Entre essas políticas, a de Promoção da Saúde tem como base o conceito ampliado de saúde, caracterizado pelo conjunto de estratégias e formas de produzir saúde que visam à equidade e qualidade de vida ${ }^{(12)}$. Essa política possui em seu escopo princípios e valores que potencializam 
a saúde de indivíduos e coletividades, reduzem vulnerabilidades e riscos à saúde, e, com o conhecimento mais abrangente dessas condições, trazem grande impacto sobre a promoção da saúde ${ }^{(6,12,13)}$.

Dessa forma, o objetivo deste estudo foi investigar a associação entre a autopercepção negativa de saúde e características individuais entre idosos de grupos comunitários do Sudeste do Brasil.

\section{MÉTODOS}

Trata-se de um estudo transversal, realizado pelo Núcleo de Estudos da Pessoa Idosa da Universidade Federal de Juiz de Fora, campus Governador Valadares, Minas Gerais, Brasil, entre os anos de 2014 e 2017.

A pesquisa foi realizada em uma amostra de conveniência de pessoas idosas participantes de 2 grupos de convivência de dispositivos sociais e/ou religiosos da cidade. Esses grupos têm como objetivo principal a promoção da saúde, por meio da prática de atividade física e o incentivo ao convívio social. A frequência dos encontros é diária e são realizadas diversas atividades coletivas, tais como: dança, jogos de tabuleiro, artesanato, natação, hidroginástica e exercícios de alongamento. Ainda, em parceria com o grupo de pesquisa da Universidade, eram realizados atendimentos semanais de projetos de extensão, como atendimentos e ações de educação em saúde sobre temas de interesse dessa população (orientações nutricionais, farmacêuticas e odontológicas), com a realização de aulas de dança, oficinas de memória e pilates.

Existem inúmeros grupos de convivência na cidade de Governador Valadares, sendo assim, o critério de inclusão dos grupos na presente pesquisa foi a relação que esses tinham com a universidade, ou seja, locais em que também são realizados atendimentos de projetos de extensão universitária. Os grupos sem qualquer vínculo com a universidade foram automaticamente excluídos. Quanto ao critério de inclusão dos participantes no presente estudo, considerou-se pessoas idosas, frequentadoras dos locais pré-estalebecidos e que apresentaram idade maior ou igual a 60 anos no dia da avaliação. Por outro lado, foram excluídos os idosos que não compareceram à entrevista após três agendamentos, os que apresentaram alguma dificuldade na comunicação, invibializando a coleta de dados, bem como os que se recusaram a participar do estudo.

Em relação ao cálculo do tamanho amostral, o território urbano do município conta com 25.905 pessoas com idade entre 60 a 80 anos $^{(14)}$. De acordo com esse dado e considerando uma margem de erro (alfa) de $5 \%$ e um nível de confiança de $90 \%$, calculou-se o tamanho amostral do presente estudo, obtendo-se o valor de 173 participantes.

Para a coleta de dados, utilizou-se um questionário estruturado pelos próprios pesquisadores, especificamente para este trabalho, baseado em questões utilizadas em inquérito populacional de abrangência nacional(15), clássicas aos estudos epidemiológicos. Pesquisadores, devidamente e igualmente treinados, coletaram os dados por meio de entrevista face a face, previamente agendada por contato telefônico, nos locais onde eram realizados os grupos de convivência. Os responsáveis de cada grupo de convivência forneceram os contatos telefônicos. Além da entrevista, também aferiram-se as medidas antropométricas de peso, altura e circunferência da cintura (CC), utilizando-se, respectivamente, balança digital portátil, com capacidade para $180 \mathrm{Kg}$ e precisão de 100g (P150M, Líder ${ }^{\circledast}$, São Paulo, Brasil), estadiômetro portátil, com capacidade de $2,13 \mathrm{~m}$ e precisão de $1 \mathrm{~mm}$ (Alturexata ${ }^{\circledR}$, Minas Gerais, Brasil), e trena antropométrica, com capacidade para $180 \mathrm{~cm}$ e precisão de $1 \mathrm{~mm}$ (Wiso ${ }^{\circledR}$, São Paulo, Brasil).

$\mathrm{Na}$ antropometria, observaram-se as técnicas preconizadas pela Organização Mundial de Saúde ${ }^{(16,17)}$, obtendo-se o peso com o participante posicionado no centro da balança, previamente zerada, descalço, ereto, com os pés juntos, braços estendidos ao longo do corpo e vestindo o mínimo de roupas. Aferiu-se a altura após inspiração da pessoa idosa e de seu posicionamento ereto no centro do estadiômetro, com a cabeça erguida, olhar fixo em um ponto na linha dos olhos, descalça, pernas paralelas e alinhamento dos calcanhares, panturrilhas, glúteos, escápulas e parte posterior da cabeça. Realizou-se a medida da CC no ponto médio entre a crista ilíaca e o rebordo costal lateral, sendo a leitura realizada após uma expiração do avaliado ${ }^{(16,17)}$.

Avaliou-se a autopercepção de saúde, variável resposta, por meio da seguinte pergunta: o(a) senhor(a) diria que sua saúde está: ruim, razoável, boa ou muito boa? Categorizaram-se as respostas em autopercepção de saúde negativa (ruim e razoável) e positiva (boa e muito boa).

As variáveis explicativas obtidas estão divididas em quatro blocos. No primeiro bloco, denominado demográfico e socioeconômico, incluíram-se as variáveis idade, sexo (feminino e masculino), estado civil (com e sem companheiro(a)), escolaridade (até 4 anos, 5 a 8 anos, 9 a 11 anos e $\geq 12$ anos de estudo) e renda ( $<2$ salários mínimos e $\geq 2$ salários mínimos). 
No segundo bloco, incluíram-se as variáveis do domínio estilo de vida: tabagismo (nunca fumou, ex-fumante e fuma atualmente) $)^{(15)}$, consumo de bebida alcoólica (sim e não) ${ }^{(15)}$, prática de atividade física no último mês (sim e não) e consumo diário de frutas e/ou hortaliças (sim e não) ${ }^{(18)}$.

No terceiro bloco, de saúde, incluíram-se as variáveis doenças crônicas (diabetes, hipertensão arterial, artrite/ reumatismo, osteoporose, depressão e/ou asma/bronquite), autorreferidas (relato de uma ou nenhuma doença crônica e relato de duas ou mais) ${ }^{(15)}$, uso de medicamento (sim e não) e capacidade funcional (independente ou dependente), mensuradas pelas perguntas: você tem dificuldade para ir da cama para cadeira ou da cadeira para a cama? Você tem dificuldade para alimentar-se sozinho(a)? Você tem dificuldade para vestir-se sozinho(a)? Você tem dificuldade para tomar banho sozinho(a)? Você tem dificuldade para caminhar pelo menos $1,5 \mathrm{~km}$ sem se cansar? As opções de resposta para essas perguntas foram sim ou não. A categorização da variável deu-se pela soma dessas perguntas, categorizadas em "dependente" - quando os indivíduos respondiam sim para, pelo menos, um item - e em "independente" - quando respondiam não para todas as perguntas. As perguntas sobre capacidade funcional foram baseadas no Índice de Katz ${ }^{(19)}$.

$\mathrm{E}$, por fim, no quarto bloco, incluíram-se as variáveis antropométricas: índice de massa corporal (IMC) (baixo peso=IMC $<22 \mathrm{~kg} / \mathrm{m} 2$; eutrófico $=I M C$ entre $22 \mathrm{~kg} / \mathrm{m} 2$ e $27 \mathrm{~kg} / \mathrm{m} 2$; e sobrepeso $=I M C>27 \mathrm{~kg} / \mathrm{m} 2)^{(20)}$ e circunferência da cintura (CC) (sem risco cardiovascular $=$ homens $\mathrm{CC}<94 \mathrm{~cm}$ e mulheres $<80 \mathrm{~cm}$; risco aumentado $=$ homens $C C$ entre 94 e $101 \mathrm{~cm}$ e mulheres entre 80 e $87 \mathrm{~cm}$; e risco muito aumentado $=$ homens $C C \geq 102 \mathrm{~cm}$ e mulheres $\geq 88 \mathrm{~cm})^{(17)}$.

Nas análises descritivas, calcularam-se as frequências e proporções para as variáveis categóricas e para a idade, devido à distribuição não paramétrica, a partir da mediana e do intervalo interquartil entre o primeiro e o terceiro quartis (1Q-3Q). Para verificar a associação entre autopercepção de saúde e as variáveis explicativas categóricas, utilizaram-se o teste qui-quadrado e o teste exato de Fisher, este quando as caselas possuíam valores esperados menor do que 5. Além disso, verificou-se a associação entre a variável resposta e a idade por meio do teste Mann Whitney. Selecionaram-se as variáveis explicativas que apresentaram valor de $p$ inferior a 0,20 na análise bivariada para a análise no modelo logístico multivariado, considerando o método de stepwise backward para a retirada das variáveis. Nessa análise, adotou-se entrada hierárquica das variáveis em blocos, com a introdução dos blocos na seguinte sequência: socioeconômico e demográfico, estilo de vida, saúde e medidas antropométricas. Com a entrada de cada bloco de variáveis, obtinha-se um novo modelo de análise. Considerou-se nível de significância de $5 \%$, com intervalo de confiança de $95 \%$ (IC 95\%), nas análises.

Esta pesquisa obteve a aprovação do Comitê de Ética em Pesquisa da Universidade Federal de Juiz de Fora, sob o Parecer n. ${ }^{\circ} 1.233 .097$, de acordo com a Resolução n. ${ }^{\circ} 466 / 2012$ e a Resolução n. ${ }^{\circ 510 / 2016}$. Todos os participantes receberam previamente informações sobre os procedimentos de coleta de dados e assinaram o Termo de Consentimento Livre e Esclarecido.

\section{RESULTADOS}

A amostra total foi constituída por 157 participantes, atingindo $90,8 \%$ do tamanho amostral calculado, pois vinte pessoas idosas não compareceram à entrevista após três agendamentos.

A proporção de autopercepção de saúde negativa foi de 32,5\% (IC95\%: 25,1 - 39,9). A mediana de idade encontrada foi de 69 anos, sendo 65 anos correspondente ao primeiro quartil e 74 ao terceiro. Quanto à amostra, em sua maioria, composta por pessoas idosas do sexo feminino (85,4\%), vivendo sem companheiro(a) $(57,3 \%)$, de baixa escolaridade $(49,7 \%)$, com renda inferior a dois salários mínimos $(56,6 \%)$ e presença de duas ou mais doenças crônicas autorreferidas (52,9\%). Demais informações sobre a caracterização da amostra estão descritas na Tabela I.

Nas análises bivariadas, a autopercepção de saúde associou-se à escolaridade $(p=0,006)$ e à renda $(p<0,001)$ no bloco das variáveis socioeconômicas e demográficas. No bloco de saúde, a autopercepção de saúde associou-se à doença autorreferida $(p<0,001)$, ao uso de medicamentos $(p=0,005)$ e à capacidade funcional $(p=0,012)$. No bloco estilo de vida, a atividade física associou-se à autopercepção de saúde $(p=0,001)$. E, finalmente, no bloco de avaliação antropométrica, a CC associou-se à autopercepção de saúde $(p=0,043)$, conforme dados apresentados na Tabela II.

De acordo com a Tabela III, os resultados da análise multivariada mostraram que, independente da idade e do sexo, pessoas idosas que possuíam renda menor que dois salários mínimos apresentaram maior chance de perceberem sua saúde como sendo negativa ( $O R=5,02$; IC95\%: 2,08 - 12,08), bem como aqueles que não praticavam atividade física (OR=3,51; IC95\%: 1,26 - 9,78). Ademais, idosos que autorreferiram duas ou mais doenças também apresentaram maior chance de perceber a sua saúde como sendo negativa (OR: 4,96; IC95\%: 2,10-11,72) em relação aos que autorreferiram nenhuma ou uma doença. O modelo apresentou ajuste adequado (Goodness off it $p=0,598$ ). 
Tabela I - Caracterização das pessoas idosas participantes de grupos comunitários de Governador Valadares, Minas Gerais, 2014-2017.

\begin{tabular}{|c|c|}
\hline Variáveis & $\begin{array}{c}\text { Total }(n=157) \\
n / \text { mediana }(\% / 1 Q-3 Q)\end{array}$ \\
\hline Idade (anos) & $69(65-74)$ \\
\hline \multicolumn{2}{|l|}{ Sexo } \\
\hline Masculino & $23(14,6)$ \\
\hline Feminino & $134(85,4)$ \\
\hline \multicolumn{2}{|l|}{ Estado civil } \\
\hline Sem companheiro(a) & $90(57,3)$ \\
\hline Com companheiro(a) & $67(42,7)$ \\
\hline \multicolumn{2}{|l|}{ Escolaridade (anos) } \\
\hline Até 4 & $77(49,7)$ \\
\hline 5 a 8 & $27(17,4)$ \\
\hline 9 a 11 & $27(17,4)$ \\
\hline$\geq 12$ & $24(15,5)$ \\
\hline \multicolumn{2}{|l|}{ Renda** $^{* *}$} \\
\hline$<2 \mathrm{SM}$ & $86(56,6)$ \\
\hline$\geq 2 \mathrm{SM}$ & $66(43,4)$ \\
\hline \multicolumn{2}{|l|}{ Morbidade } \\
\hline 1 ou nenhuma doença & $74(47,1)$ \\
\hline$\geq 2$ doenças & $83(52,9)$ \\
\hline \multicolumn{2}{|l|}{ Uso de medicamentos } \\
\hline Não & $14(8,9)$ \\
\hline Sim & $143(91,1)$ \\
\hline \multicolumn{2}{|l|}{ Capacidade funcional $^{* * *}$} \\
\hline Independente & $97(69,8)$ \\
\hline Dependente & $42(30,2)$ \\
\hline \multicolumn{2}{|l|}{ Tabagismo ${ }^{\star * * *}$} \\
\hline Nunca fumou & $101(65,6)$ \\
\hline Ex-fumante & $46(29,9)$ \\
\hline Fumante & $7(4,5)$ \\
\hline \multicolumn{2}{|l|}{ Atividade física } \\
\hline Não & $26(16,7)$ \\
\hline Sim & $130(83,3)$ \\
\hline \multicolumn{2}{|l|}{ Consumo de álcool } \\
\hline Não & $93(67,4 \%)$ \\
\hline Sim & $45(32,6)$ \\
\hline \multicolumn{2}{|l|}{ Consumo saudável } \\
\hline Não & $8(5,1)$ \\
\hline Sim & $149(94,9)$ \\
\hline \multicolumn{2}{|l|}{ IMC } \\
\hline Eutrófico & $54(34,4)$ \\
\hline Baixo peso & $14(8,9)$ \\
\hline Sobrepeso & $89(56,7)$ \\
\hline \multicolumn{2}{|l|}{ CC } \\
\hline Sem risco & $39(24,8)$ \\
\hline Risco aumentado & $51(32,5)$ \\
\hline Risco muito aumentado & $67(42,7)$ \\
\hline
\end{tabular}

1Q-3Q: intervalo entre o primeiro e o terceiro quartis; SM: salário mínimo; IMC: índice de massa corporal; CC: circunferência de cintura. "02 missing; ${ }^{* *} 05$ missing; ${ }^{* *} 18$ missing; ${ }^{* * *} 03$ missing; ${ }^{* * * *} 01$ missing; ${ }^{* * * * * *} 19$ missing 
Tabela II - Associação entre autopercepção de saúde e as variáveis investigadas entre pessoas idosas participantes de grupos comunitários de Governador Valadares, Minas Gerais, 2014-2017.

\begin{tabular}{|c|c|c|c|}
\hline Variáveis & $\begin{array}{c}\text { Autopercepção negativa } \\
\text { n/ mediana (\% / 1Q-3Q) }\end{array}$ & $\begin{array}{c}\text { Autopercepção positiva } \\
\text { n/ mediana (\% / 1Q-3Q) }\end{array}$ & $p$-valor \\
\hline Idade (anos) & $69(65-75)$ & $69(64-74)$ & 0,680 \\
\hline Sexo & & & 0,478 \\
\hline Masculino & $6(11,8)$ & $17(16,0)$ & \\
\hline Feminino & $45(88,2)$ & $89(84,0)$ & \\
\hline Estado civil & & & 0,792 \\
\hline Sem companheiro(a) & $30(58,8)$ & $60(56,6)$ & \\
\hline Com companheiro(a) & $21(41,2)$ & $46(43,4)$ & \\
\hline Escolaridade & & & $0,006^{*}$ \\
\hline Até 4 anos & $29(59,2)$ & $48(45,3)$ & \\
\hline 5 a 8 anos & $13(26,5)$ & $14(13,2)$ & \\
\hline 9 a 11 anos & $4(8,2)$ & $23(21,7)$ & \\
\hline$\geq 12$ anos & $3(6,1)$ & $21(19,8)$ & \\
\hline Renda & & & $<0,001^{*}$ \\
\hline$<2 \mathrm{SM}$ & $39(78,0)$ & $47(46,1)$ & \\
\hline$\geq 2 \mathrm{SM}$ & $11(22,0)$ & $55(53,9)$ & \\
\hline Morbidade & & & $<0,001^{*}$ \\
\hline 1 ou nenhuma doença & $12(23,5)$ & $62(58,5)$ & \\
\hline$\geq 2$ doenças & $39(76,5)$ & $44(41,5)$ & \\
\hline Uso de medicamentos & & & $0,005^{* *}$ \\
\hline Não & $0(0,0)$ & $14(13,2)$ & \\
\hline $\operatorname{Sim}$ & $51(100,0)$ & $92(86,8)$ & \\
\hline Capacidade funcional & & & $0,012^{*}$ \\
\hline Independente & $27(56,3)$ & $70(76,9)$ & \\
\hline Dependente & $21(43,8)$ & $21(23,1)$ & \\
\hline Tabagismo & & & 0,453 \\
\hline Nunca fumou & $30(58,8)$ & $71(68,9)$ & \\
\hline Ex-fumante & $18(35,3)$ & $28(27,2)$ & \\
\hline Fumante & $3(5,9)$ & $4(3,9)$ & \\
\hline Atividade física & & & $0,001^{*}$ \\
\hline Não & $16(31,4)$ & $10(9,5)$ & \\
\hline Sim & $35(68,6)$ & $95(90,5)$ & \\
\hline Consumo de álcool & & & 0,076 \\
\hline Não & $37(77,1)$ & $56(62,2)$ & \\
\hline Sim & $11(22,9)$ & $34(37,8)$ & \\
\hline Consumo saudável & & & 0,276 \\
\hline Não & $4(7,8)$ & $4(3,8)$ & \\
\hline $\operatorname{Sim}$ & $47(92,2)$ & $102(96,2)$ & \\
\hline IMC & & & 0,205 \\
\hline Eutrófico & $14(27,5)$ & $40(37,7)$ & \\
\hline Baixo peso & $3(5,9)$ & $11(10,4)$ & \\
\hline Sobrepeso & $34(66,7)$ & $55(51,9)$ & \\
\hline CC & & & $0,043^{*}$ \\
\hline Sem risco & $9(17,6)$ & $30(28,3)$ & \\
\hline Risco aumentado & $13(25,5)$ & $38(35,8)$ & \\
\hline Risco muito aumentado & $29(56,9)$ & $38(35,8)$ & \\
\hline
\end{tabular}

1Q-3Q: intervalo entre o primeiro e o terceiro quartis; SM: salário mínimo; IMC: índice de massa corporal; CC: circunferência de cintura 
Tabela III - Análise multivariada por meio de regressão logística para a autopercepção negativa de saúde de pessoas idosas participantes de grupos comunitários de Governador Valadares, Minas Gerais, 2014-2017.

\begin{tabular}{lccc}
\hline Variáveis & OR & IC 95\% & $p$-valor \\
\hline Renda & & & \\
$\quad<2$ SM & 5,02 & $2,08-12,08$ & \\
$\quad \geq 2$ SM & 1,00 & & \\
$\quad$ Atividade física & & 1,001 & \\
$\quad$ Não & 3,51 & & \\
$\quad$ Sim & 1,00 & & \\
Morbidade & & $2,10-11,72$ & $<0,001$ \\
$\quad \geq 2$ doenças & 4,96 & & \\
$\quad$ ou nenhuma doença & 1,00 & & \\
\hline
\end{tabular}

"Modelo ajustado por idade e sexo. OR: odds ratio; IC 95\%: intervalo de confiança de 95\%; SM: salário mínimo

\section{DISCUSSÃO}

Os principais achados do presente estudo indicaram que as pessoas idosas que relataram uma autopercepção negativa de saúde tinham menor renda, não praticavam atividade física e autorreferiram duas ou mais doenças crônicas.

No presente estudo, 32,5\% dos idosos apresentaram autopercepção negativa de saúde, enquanto na literatura a ocorrência dessa condição em idosos residentes na comunidade varia entre $12,6 \%$ a 70,1\%(4,12,21-23). Essa amplitude pode ser explicada, em parte, pela ausência de padrão internacional de opções de respostas e de categorização(21). Outras questões também podem explicar essas diferenças, como a fonte de informação (pessoa entrevistada ou terceiros, como cuidador ou responsável), as diferenças no posicionamento da questão no questionário, e aspectos do delineamento dos estudos e ajustes na análise ${ }^{(24)}$. As comparações entre estudos, países e subpopulações devem ser realizadas com cautela, uma vez que as diferenças entre as prevalências podem não ser coerentes com os indicadores objetivos, e sim atribuídas às diferenças metodológicas ${ }^{(25)}$.

A autopercepção negativa de saúde apresentou característica multidimensional no grupo avaliado, pois esteve associada às condições socioeconômicas, de estilo de vida e de saúde, característica detectada também em outros estudos, demonstrando aproximação de diferentes determinantes com o conceito ampliado de saúde e corroborando com os achados aqui apresentados ${ }^{(21,24,26-29)}$.

A renda e as condições de vida se relacionam fortemente às suas condições de saúde e a autopercepção dela pelo idoso ${ }^{(4,25,30)}$. Sabe-se que a pobreza e/ou baixos níveis de escolaridade na velhice se traduzem em menor acesso aos serviços, pior adesão às intervenções preventivas e curativas, maiores gastos proporcionais do rendimento com medicamentos e alimentação, redução do convívio social e maior nível de dependência do indivíduo ${ }^{(30,31)}$.

Quanto ao estilo de vida, a prática de atividade física traz diversos benefícios a esse grupo especificamente, tais como: melhora da funcionalidade biológica e preservação orgânica; controle do peso corporal e massa gorda; preservação da massa muscular; prevenção e controle de doenças; manutenção da capacidade funcional e autonomia $^{(32)}$. Ademais, essa prática favorece a formação de redes de socialização e maior interação com seus pares e com outras gerações, o que proporciona a troca de experiências, conhecimentos e inclusão social, resultando na melhora da autoestima e da saúde ${ }^{(33,34)}$. Cabe ressaltar que, entre os fatores relacionados ao comportamento e estilo de vida, a prática regular de atividade física é um dos mais importantes determinantes do envelhecimento ativo na população( ${ }^{(35)}$.

Evidências sobre a associação entre a autopercepção de saúde e a prática de atividade física podem ser verificadas em diversos estudos, nacionais e internacionais. Em uma investigação conduzida com pessoas idosas usuárias do Sistema Único de Saúde no município de Goiânia, Goiás, identificaram-se alguns fatores associados à autopercepção negativa do estado de saúde, como a baixa escolaridade, o uso de cinco ou mais medicamentos, a perda de peso recente e não praticar atividade física, sendo o último fortemente associado a essa condição ${ }^{(26)}$. Da mesma forma, em outro estudo, realizado na Região Metropolitana de Belo Horizonte, verificou-se a associação entre a redução da prática de atividade física e a autopercepção negativa do estado de saúde entre as pessoas idosas $^{(28)}$. E, finalmente, um estudo recente mostrou que idosos, residentes em um país europeu, que nunca praticaram atividade física vigorosa ou moderada, perceberam sua saúde como sendo negativa ${ }^{(36)}$. 
Além dos estudos acima mencionados, que investigaram a atividade física no lazer, um recente estudo, desenvolvido em países da Europa e nos Estados Unidos, com 15.333 idosos e 20.907 idosas, também investigou a relação entre a autopercepção de saúde e a atividade física no trabalho doméstico. Seus resultados mostraram que existe associação entre autopercepção de saúde e a realização desse tipo de atividade, evidenciando que quanto maior o tempo de prática de atividade doméstica, maior é a chance dos indivíduos perceberem sua própria saúde como boa(23).

Em relação à presença de doenças crônicas, os resultados do presente estudo corroboram outras pesquisas. Um estudo desenvolvido na China, com adultos e pessoas idosas, demonstrou que a presença de múltiplas e variadas doenças esteve associada à autopercepção negativa de saúde, sendo as doenças cardiometabólicas as que apresentaram maior força de associação na população investigada ${ }^{(37)}$. Uma revisão de literatura realizada demonstrou que a autopercepção negativa de saúde é predominante entre as pessoas idosas, estando associada prioritariamente à presença de doenças ${ }^{(22)}$. Ademais, nesta revisão, a autopercepção negativa de saúde associou-se ao número de medicamentos em uso, à renda familiar, à ocorrência de internações, à consultas médicas, à dificuldade/ incapacidade para atividades de vida diária, à presença de sintomas depressivos, à ansiedade e à queixa de insônia ${ }^{(22)}$.

A presença de doenças, comumente observadas nesse grupo populacional, está associada à autopercepção de saúde, pois constitui fator relevante ao aparecimento ou desenvolvimento de incapacidades funcionais e instrumentais de vida diária e, consequentemente, afeta a qualidade de vida e o bem-estar desses indivíduos ${ }^{(37-39)}$. No entanto é importante destacar que, muitas vezes, a autopercepção de saúde boa/muito boa ocorre mesmo entre aqueles com diagnóstico de doenças crônicas. Esse fato reforça a ideia de que o julgamento de sua própria condição de saúde está mais intimamente relacionado à ocorrência de incapacidades e perda de autonomia do que à própria doença( ${ }^{(6)}$.

Este estudo, no entanto, não encontrou associação entre a capacidade funcional e a autopercepção negativa de saúde. Esse fato pode estar relacionado ao processo de seleção da amostra e à sua caracterização, pois, como foram selecionadas pessoas idosas que participavam ativamente de grupos comunitários, espera-se que sejam mais independentes, não sendo possível identificar um número de participantes dependentes suficiente para verificar uma possível associação. Por outro lado, a literatura científica aponta que essa associação pode existir. Um estudo realizado com esse grupo populacional residente em três cidades brasileiras demostrou que a incapacidade funcional esteve associada à autopercepção negativa de saúde ${ }^{(21)}$. Uma pesquisa realizada na Coréia do Sul avaliou a capacidade preditiva da autopercepção negativa da saúde para o declínio de capacidade funcional após dois anos de acompanhamento de 2.824 pessoas idosas sem incapacidades na linha de base. Os resultados demonstraram que, ao final do acompanhamento, $4,9 \%$ dos participantes relataram um declínio da capacidade funcional, e a autopercepção negativa de saúde esteve significativamente associada a esse declínio ${ }^{(40)}$.

É importante ressaltar que outros fatores podem estar associados à autopercepção negativa de saúde, como a baixa escolaridade, o uso de medicamentos e o consumo de bebida alcoólica ${ }^{(4,29)}$, embora isso não tenha ocorrido no presente estudo. Ademais, suporte e interação social, acesso e utilização de serviços de saúde, utilização de internet e quedas também podem estar associadas à essa condição ${ }^{(4,29)}$, mas essas variáveis não foram aqui investigadas.

Algumas limitações deste trabalho devem ser consideradas. Entre elas, o delineamento seccional do estudo, que não permite estabelecer relações de causalidade entre as variáveis explicativas estudadas e a variável resposta. Outra limitação é a presença de possível viés de seleção por se tratar de uma amostra de conveniência, em sua maioria mulheres. Ademais, o trabalho foi desenvolvido com um público participante de grupos comunitários destinados às pessoas com idade igual ou superior a 60 anos, de forma que eram expostos regularmente às ações de educação em saúde, além de convivência grupal, que pode proporcionar maior socialização e suporte para o enfrentamento de problemas. Portanto, os resultados deste estudo talvez não possam ser extrapolados para pessoas idosas da comunidade em geral e para a população masculina. $O$ uso de medidas autorreferidas pode deixar os resultados suscetíveis à influência de fatores comportamentais, culturais e sociais ${ }^{(24)}$.

Além disso, apesar dos resultados deste estudo demonstrarem associação entre a autopercepção de saúde e a prática de atividade física, nenhum questionário traduzido e validado para esse grupo específico ou equipamento foi utilizado para medir o nível de prática de atividade física, não se utilizando os parâmetros descritos na literatura para definir se o indivíduo é ou não ativo fisicamente. Quanto à capacidade funcional, também não foi utilizado nenhum instrumento traduzido e validado para medir essa variável.

Finalmente, é importante descatar que, apesar da pergunta utilizada para medir a autopercepção de saúde não ser validada para a população brasileira, tampouco para a população idosa, ela tem atraído a atenção de pesquisadores no Brasil e no mundo, uma vez que apresenta uma evidência acumulada de sua associação com mortalidade, é simples de ser apliacada e de baixo custo, além de ser facilmente compreendida pelos participantes ${ }^{(8-10)}$. Essas características do instrumento são especialmente importantes no contexto brasileiro, onde $42,3 \%$ da população tem 
menos de oito anos de estudo(3), havendo um percentual ainda maior entre as pessoas idosas. Portanto, apesar da ausência de validação no país, essa pergunta é considerada uma medida robusta de autopercepção de saúde e seu uso é fortemente aceitável nesse cenário. No entanto a questão utilizada neste estudo possuía apenas quatro opções de reposta (ruim, razoável, boa ou muito boa), enquanto outras pesquisas utilizam cinco opções de resposta (muito ruim, ruim, razoável, boa ou muito boa), fato que dificulta a comparação entre os achados dos estudos.

Apesar das limitações apresentadas, os resultados possuem validação interna, pois foram investigados $93,3 \%$ das pessoas cadastradas nos referidos grupos comunitários, os quais estavam localizados em diferentes regiões do município, com características socioeconômicas diversas, além da equipe de coleta de dados ter sido amplamente treinada e calibrada previamente e ao longo da pesquisa.

\section{CONCLUSÃO}

Os achados da pesquisa trazem informações relevantes para a literatura científica, haja vista que a autopercepção negativa de saúde associou-se à menor renda familiar, à inatividade física e à presença de duas ou mais doenças associadas. Esses resultados podem ser úteis na comparação entre dados de amostras populacionais, visto que representam um grupo de pessoas idosas ativas e inseridas na sociedade, o que vem sendo estimulado e preconizado para a melhoria da qualidade de vida nessa faixa etária. Os resultados reforçam, ainda, que a autopercepção de saúde é um construto multidimensional, influenciada por fatores individuais (alguns modificáveis), indicando a necessidade de estratégias para aumentar a adoção de hábitos e comportamentos de vida mais saudáveis.

\section{CONFLITOS DE INTERESSE}

Não houve conflitos de interesses na realização do presente estudo.

\section{FONTES DE FINANCIAMENTO}

FAPEMIG (Fundação de Amparo à Pesquisa do Estado de Minas Gerais), processo no APQ 00133-14.

CNPq (Conselho Nacional de Desenvolvimento Científico e Tecnológico), processo no 432314/2016-4.

\section{CONTRIBUIÇÕES}

Todos os autores contribuíram com a elaboração e delineamento do estudo; a aquisição, análise e interpretação de dados; e a redação e/ou revisão do manuscrito.

\section{REFERÊNCIAS}

1. Miranda GMD, Mendes ACG, Silva ALA. O envelhecimento populacional brasileiro: desafios e consequências sociais atuais e futuras. Rev Bras Geriatr Gerontol. 2016;19(3):507-59.

2. Barros MBA, Goldbaum M. Desafios do envelhecimento em contexto de desigualdade social. Rev Saúde Pública. 2018;52(Supl 2):1s.

3. Instituto Brasileiro de Geografia e Estatística. Sinopse do Censo Demográfico 2010 [Internet]. Rio de Janeiro: IBGE; 2011 [acesso em 2019 Jan 14]. Disponível em: http://biblioteca.ibge.gov.br/visualizacao/ livros/liv49230.pdf

4. Santos JLF, Duarte YAO, Lebrao, ML. Early life conditions and current health status as per the study "Health, Well-being and Aging" (SABE). Rev Bras Epidemiol. 2018;21(2):1-15.

5. Veras RP, Oliveira M. Envelhecer no Brasil: a construção de um modelo de cuidado. Ciênc Saúde Colet. 2018;23(6):1929-36.

6. Ferreira ACS, Mattos M. Atenção multiprofissional ao idoso em condição crônica na Estratégia Saúde da Família. Rev Bras Promoç Saúde. 2018;31(3):1-10.

7. Costa MR. Padrão e autopercepção alimentar de mulheres fisicamente ativas [dissertação]. Salvador: UFBA; 2013. 
8. Lindemann IL, Reis NR, Mintem GC, Sassi RAM. Autopercepção da saúde entre adultos e idosos usuários da Atenção Básica de Saúde. Ciênc Saúde Colet [Internet]. 2019 [acesso em 2019 Jan 14];24(1):45-52. doi: 10.1590/1413-81232018241.34932016

9. Falk H, Skoog I, Johansson L, Guerchet M, Mayston R, Hörder H, et al. Selfrated health and its association with mortality in older adults in China, India and Latin America-a 10/66 Dementia Research Group study. Age Ageing. 2017;46(6):932-9.

10. Inuzuka S, Jardim PCV, Abrahams-Gessel S, Souza LG, Rezende AC, Perillo NB, et al. Self-rated health status and illiteracy as death predictors in a Brazilian cohort. PLoS ONE. 2018;13(7):e0200501.

11. Ribeiro EG, Matozinhos FP, Guimarães GL, Couto AM, Azevedo RS, Mendonza IYQ. Autopercepção de saúde e vulnerabilidade clínico-funcional de idosos de Belo Horizonte/Minas Gerais. Rev Bras Enferm. 2018;71(Supl.2):860-7.

12. Ministério da Saúde (BR), Secretaria de Vigilância em Saúde, Secretaria de Atenção em Saúde. Política Nacional de Promoção da Saúde: PNPS: Anexo I da Portaria de Consolidação $n^{\circ} 2$, de 28 de setembro de 2017, que consolida as normas sobre as políticas nacionais de saúde do SUS. Brasília: Ministério da Saúde; 2018.

13. Mendes EV. The care for chronic conditions in primary health care. Rev Bras Promoç Saúde. 2018;31(2):1-3.

14. IBGE. Tabela 1378: população residente, por situação do domicílio, sexo e idade, segundo a condição no domicílio e compartilhamento da responsabilidade pelo domicílio [Internet]. [2011] [acesso em 2019 Nov 12]. Disponível em: https://sidra.ibge.gov.br/Tabela/1378\#resultado

15. Ministério da Saúde (BR), Secretaria de Vigilância em Saúde, Departamento de Vigilância de Doenças e Agravos não Transmissíveis e Promoção da Saúde. Vigitel Brasil 2014: vigilância de fatores de risco e proteção para doenças crônicas por inquérito telefônico. Brasília: Ministério da Saúde; 2015.

16. World Health Organization. Physical status: the use and interpretation of antropometry. WHO Technical Report Series. Geneva: WHO; 1995.

17. World Health Organization. Obesity: preventing and managing the global epidemic. Geneva: WHO; 1998.

18. Silvani J, Buss C, Pena GG, Recchi AF, Wendland EM. Consumo alimentar de usuários do Sistema Único de Saúde segundo o tipo de assistência e participação no Bolsa Família. Cienc Saúde Colet. 2018;23(8):2599608.

19. Duarte YAO, Andrade CL, Lebrão ML. O Índex de Katz na avaliação da funcionalidade dos idosos. Rev Esc Enferm USP. 2007;41(2):317-25.

20. Lipschitz DA. Screening for nutritional status in the elderly. Prim Care. 1994;21(1):55-67.

21. Medeiros SM, Silva LSR, Carneiro JA, Ramos GCF, Barbosa ATF, Caldeira AP. Fatores associados à autopercepção negativa da saúde entre idosos não institucionalizados de Montes Claros, Brasil. Ciênc Saúde Colet. 2016;21(11):3377-86.

22. Pagotto V, Bachion MM, Silveira EA. Autoavaliação da saúde por idosos brasileiros: revisão sistemática da literatura. Rev Panam Salud Publica. 2013;33(4):302-10.

23. Adjei NK, Brand T. Investigating the associations between productive housework ativities, sleep hours and self-reported health among elderly men and women in western industrialised countries. BMC Public Health. 2018;18(1):110.

24. Meireles AL, Xavier CC, Andrade ACDS, Friche AADL, Proietti FA, Caiaffa WT. Self-rated health in urban adults, perceptions of the physical and social environment, and reported comorbidities: the BH Health Study. Cad Saude Publica. 2015;31(Supl 1):120-35.

25. Medeiros PA, Cembranel F, Figueiró TH, Souza BB, Antes DL, Silva DAS, et al. Prevalência e simultaneidade de fatores de risco cardiovasculares em idosos participantes de um estudo de base populacional no sul do Brasil. Rev Bras Epidemiol. 2019;2(2):1-16.

26. Pagotto $V$, Nakatani AYK, Silveira EA. Fatores associados à autoavaliação de saúde ruim em idosos usuários do Sistema Único de Saúde. Cad Saude Publica. 2011;27(8):1593-60. 
27. Borim FSA, Barros MBA, Neri AL. Autoavaliação da saúde em idosos: pesquisa de base populacional no Município de Campinas, São Paulo, Brasil. Cad Saude Publica. 2012;28(4):769-80.

28. Carvalho FF, Santos JN, Souza LM, Souza NRM. Análise da percepção do estado de saúde dos idosos da região metropolitana de Belo Horizonte. Rev Bras Geriatr Gerontol. 2012;15(2):285-93.

29. Confortin SC, Giehl MWC, Antes DL, Schneider IJC, D'orsi E. Autopercepção positiva de saúde em idosos: estudo populacional no Sul do Brasil. Cad Saude Publica. 2015;31(5):1049-60.

30. Kosilov K, Kuzina I, Kosilova L, Gainullina Y, Kuznetsov V, Ivanovskaya M, et al. Health-related quality of life and socioeconomic status of imprisoned middle-age and elderly persons. Soc Theory Health. 2019;17:75-88.

31. Stenholm S, Westerlund H, Head J, Hyde M, Kawächi I, Pentti J, et al. Comorbidity and functional trajectories from midlife to old age: the Health and Retirement Study. J Gerontol A Biol Sci Med Sci. 2015;70(3):332-8.

32. Arantes RC. Diretrizes para gestão do envelhecimento ativo e com qualidade de vida para idosos brasileiros. In: Faria L, Calábria LK, Alves WA. Envelhecimento: um Olhar Interdisciplinar. São Paulo: Hucitec; 2016. p. 188-214.

33. Castro APS, Will GB, Castro MR, Ximenes CF, Cordeiro MS. Viviendo en comunidad, envejeciendo de forma saludable. Enf Global. 2019;19(1):302-45.

34. Oliveira AG, Abreu SSS, Macedo MASS, Duarte SFP, Reis LA, Lima PV. Grupos de convivência como suporte na prevenção da depressão em idosos. Rev Enferm Contemp. 2019;8(1):17-24.

35. Bauman A, Merom D, Bull FC, Buchner DM, Singh MAF. Updating the Evidence for Physical Activity: summative reviews of the epidemiological evidence, prevalence, and interventions to promote "Active Aging". Gerontol. 2016;56(Supl 2):268-80.

36. Abuladze L, Kunder N, Lang K, Vaask S. Associations between self-rated health and health behaviour among older adults in Estonia: a cross-sectional analysis. BMJ Open. 2017;7(6):e013257.

37. Song X, Wu J, Yu C, Dong W, Lv J, Guo Y, et al. Association between multiple comorbidities and self-rated health status in middle-aged and elderly Chinese: the China Kadoorie Biobank study. BMC Public Health. 2018;18(744):1-24.

38. Axelsson $\AA \AA B$, Sunnerhagen KS, Herlitz J. Representativity and co-morbidity: two factors of importance when reporting health status among survivors of cardiac arrest. Resuscitation. 2016;101:44-9.

39. Gavasso WC, Beltrame V. Functional capacity and reported morbidities: a comparative analysis in the elderly. Rev Bras Geriatr Gerontol. 2017;20(3):398-408.

40. Kim SH, Cho B, Won CW, Hong YH, Son KY. Self-reported health status as a predictor of functional decline in a community-dwelling elderly population: nationwide longitudinal survey in Korea. Geriatr Gerontol Int. 2016;17(6):885-92.

\author{
Endereço para correspondência: \\ Camila Teixeira Vaz \\ Universidade Federal de Juiz de Fora - Campus Governador Valadares \\ Rua São Paulo, 745 \\ Bairro: Centro \\ CEP: 35010-180 - Governador Valadares - MG - Brasil \\ E-mail: milatvaz@yahoo.com.br
}

Como citar: Vaz CT, Almeida NAV, Kelmann RG, Queiroz ACC, Barbosa MCA, Silva CLA. Fatores associados à autopercepção de saúde entre idosos de grupos comunitários. Rev Bras Promoç Saúde. 2020;33:10328. 